\title{
Asymptotic estimation theory for a finite dimensional pure state model
}

\author{
Masahito Hayashi \\ Department of Mathematics, Kyoto University, Kyoto 606-01, Japan \\ e-mail address: masahito@kusm.kyoto-u.ac.jp
}

\begin{abstract}
The author studies the optimization of measurement for $n$ copies of pure states. The asymptotic efficiency of the optimal measurement is calculated in the large deviation sense.
\end{abstract}

\section{Introduction}

Recently, relating to researches of optical communications, quantum optics and quantum computer, there has been more and more necessity of researches for statistical estimation of quantum states [1]-[3]. However, we have few mathematical rigorous formulations about a statistical estimation for quantum states. Helstrom developed a general local estimation theory for one-parameter families [4][5].

To the author's knowledge, there are only three models of multi-parameter family whose attainable Cramér-Rao type bound is explicitly derived; the quantum Gaussian model [5][6], the spin $1 / 2$ system three-parameter model [7]-[10] and the pure state model [10][11].

There exist many papers about quantum state reconstruction and quantum tomography [16]. In these papers, some consistent estimators (consistent measurements) are constructed. But, These papers don't describe the optimization of consistent estimators with respect to the asymptotic efficiency of estimation in the large deviation sense.

We have few asymptotic optimal estimation theories for quantum states. Nagaoka established a local asymptotic estimation theory for one-parameter families [12]. We need a global asymptotic estimation theory for quantum states families.

In this paper, the asymptotic optimal measurements and the minimal error are derived under the assumption that the object state is a pure state and that the dimension of the Hilbert space which corresponds to the physical system of interest is finite.

Let us explain briefly the contents of the present paper. In section 2, the quantum i.i.d. condition is introduced. This condition is important for the following discussions.

In section 3, Holevo's theory for covariant families and covariant measurements is summarized [5][13]. Mackey established the covariant measurement theory with respect to an action of a group [15]. Holevo proved the quantum Hunt-Stein theorem [13]. These formulations are necessary for the following sections.

In section 4 , the optimal measurement under the quantum i.i.d. condition is derived. The optimal measurement is independent of the choice of deviation measure satisfying the natural condition. 
In section 5, the optimal deviations of the square of distances is calculated. In section 6, the global large deviation is evaluated.

In section 0, we evaluate the gap between the classical statistical estimation (the classical maximum likelihood estimation) based on $n$ data of the optimal measurement on 1-particle system and the optimal measurement on $n$-particle system ( $n$-i.i.d. model). Actually, the gap vanishes in the sense of large deviation on this model.

\section{Pure state $n$-i.i.d. model}

Let $\mathcal{H}:=\mathbf{C}^{k}$ be a Hilbert space which corresponds to the physical system of interest, and let $\mathcal{P}(\mathcal{H}), \mathcal{S}(\mathcal{H})$ be the set of pure states on $\mathcal{H}$, the set of density operators on $\mathcal{H}$ respectively. In this paper, we consider measurements whose measurable set is $\mathcal{P}(\mathcal{H})$.

The purpose of this paper is finding the optimal measurement to estimate a quantum pure state in a family.

For a pure state $\rho \in \mathcal{P}(\mathcal{H})$, a possible measurement is represented by an element of $\mathcal{M}(\mathcal{P}(\mathcal{H}), \mathcal{H})$, where $\mathcal{M}(\Omega, \mathcal{H})$ denotes generalized measurements (positive operator valued measures i.e. POMs) whose measurable set is $\Omega$ on $\mathcal{H}$. (We let the $\sigma$-field be the Borel sets. See [4][5].)

Definition 2.1 Fubini-Study distance $d_{f s}$ (which is the geodesic distance of Fubini-Study metric) is defined as:

$$
\cos d_{f s}(\rho, \hat{\rho})=\sqrt{\operatorname{tr} \rho \hat{\rho}}, 0 \leq d_{f s}(\rho, \hat{\rho}) \leq \frac{\pi}{2} .
$$

Bures's distance $d_{b}$ is defined in the usual way:

$$
d_{b}(\rho, \hat{\rho}):=\sqrt{1-\operatorname{tr} \rho \hat{\rho}}
$$

Let $W(\rho, \hat{\rho})$ be a measure of deviation of the measured value $\hat{\rho}$ from the actual value $\rho$.

Lemma 2.1 For a measure of deviation, the following are equivalent.

○ $W(\rho, \hat{\rho})=W\left(g \rho g^{*}, g \hat{\rho} g^{*}\right)$ for $g \in \mathrm{SU}(k), \rho, \hat{\rho} \in \mathcal{P}(\mathcal{H})$.

- There exists a function $h$ on $[0,1]$ such that $W(\rho, \hat{\rho})=h \circ d_{f s}(\rho, \hat{\rho})$

It is natural to assume that a deviation measure $W(\rho, \hat{\rho})$ is monotone increasing with respect to Fubini-Study distance $d_{f s}$. Let $\mathcal{H}_{1}, \ldots, \mathcal{H}_{n}$ be $n$ Hilbert spaces which correspond to the physical systems. Then their composite system is represented by the tensor Hilbert space:

$$
\mathcal{H}^{(n)}:=\mathcal{H}_{1} \otimes \cdots \otimes \mathcal{H}_{n}=\bigotimes_{i=1}^{n} \mathcal{H}_{i}
$$

Thus, a state on the composite system is denoted by a density operator $\rho$ on $\mathcal{H}^{(n)}$. In particular if $n$ element systems $\left\{\mathcal{H}_{i}\right\}$ of the composite system $\mathcal{H}^{(n)}$ are independent of each other, there exists a density $\rho_{i}$ on $\mathcal{H}_{i}$ such that

$$
\rho^{(n)}=\rho_{1} \otimes \cdots \otimes \rho_{n}=\bigotimes_{i=1}^{n} \rho_{i} .
$$


The condition:

$$
\mathcal{H}_{1}=\cdots=\mathcal{H}_{n}=\mathcal{H}, \rho_{1}=\cdots=\rho_{n}=\rho
$$

corresponds to the independent and identically distributed condition (i.i.d. condition) in the classical case. In this paper, we consider under this condition (3) called the quantum i.i.d. condition. The model $\{\rho^{(n)}=\underbrace{\rho \otimes \cdots \otimes \rho}_{n} \mid \rho \in \mathcal{P}(\mathcal{H})\}$ is called $n$-i.i.d. model. As $\rho$ is a pure state, $\mathcal{H}^{(n)}$ and $\rho^{(n)}$ are simplified as follows. Letting $\rho=|\phi\rangle\langle\phi| \in \mathcal{P}(\mathcal{H})$, we have

$$
\rho^{(n)}=\left|\phi^{(n)}\right\rangle\left\langle\phi^{(n)}\right|, \phi^{(n)}:=\overbrace{\phi \otimes \ldots \otimes \phi}^{n} .
$$

The vector $\phi^{(n)}$ is included in $n$-th symmetric tensor space. The state family $\left\{\rho^{(n)} \mid \rho \in\right.$ $\mathcal{P}(\mathcal{H})\}$ is regarded a state family on $n$-th symmetric tensor space. Denoting the $n$ th symmetric tensor space on $\mathcal{H}$ by $\mathcal{H}_{s}^{(n)}$, all of possible measurements are represented elements of $\mathcal{M}\left(\mathcal{P}(\mathcal{H}), \mathcal{H}_{s}^{(n)}\right)$. The mean error of the measurement $\Pi \in \mathcal{M}\left(\mathcal{P}(\mathcal{H}), \mathcal{H}_{s}^{(n)}\right)$ with respect to a deviation measure $W(\rho, \hat{\rho})$, provided that the actual state is $\rho$, is equal to

$$
\mathcal{D}_{\rho}^{W,(n)}(\Pi):=\int_{\mathcal{P}(\mathcal{H})} W(\rho, \hat{\rho}) \operatorname{tr}\left(\Pi(d \hat{\rho}) \rho^{(n)}\right)
$$

In minimax approach the maximum possible error measure $W(\rho, \hat{\rho})$

$$
\mathcal{D}^{W,(n)}(\Pi):=\max _{\rho \in \mathcal{P}(\mathcal{H})} \mathcal{D}_{\rho}^{W}(\Pi)
$$

is minimized.

\section{Quantum Hunt-Stein theorem}

In this section, the quantum Hunt-Stein theorem established by Holevo[5][13] is summarized. Let $G$ be a compact transitive Lie group of all transformations on a compact parametric set $\Theta$, and $\left\{V_{g}\right\}$ a continuous unitary irreducible representation of $G$ in a finite-dimensional Hilbert space $\mathcal{H}^{\prime}:=\mathbf{C}^{k^{\prime}}$, and $\mu$ a $\sigma$-finite invariant measure on group $G$ such that $\mu(G)=1$.

Definition 3.1 A measurement $\Pi \in \mathcal{M}\left(\Theta, \mathcal{H}^{\prime}\right)$ is covariant with respect to $\left\{V_{g}\right\}$ if

$$
V_{g}^{*} \Pi(B) V_{g}=\Pi\left(B_{g^{-1}}\right)
$$

for any $g \in G$ and any Borel $B \subset \Theta$, where

$$
B_{g}:=\{g \theta \mid \theta \in B\}
$$

$\mathcal{M}_{V}(\Theta)$ denotes the set of covariant measurements with respect to $\left\{V_{g}\right\}$. 
Theorem 3.1 For any $\theta \in \Theta$, the map $V^{\theta}: \mathcal{S}(\mathcal{H}) \rightarrow \mathcal{M}_{V}(\Theta)$ is surjective. For $P \in$ $\mathcal{S}(\mathcal{H}), V^{\theta}(P)$ is defined as follows:

$$
V^{\theta}(P)(B):=k^{\prime} \int_{\{g \theta \in B\}} V_{g} P V_{g}^{*} \mu(d g) \text { for } B \in \mathcal{B}(\Theta) .
$$

Let $\theta$ be a parameter specifying some aspects of the state preparation, so there is a family $\left\{S_{\theta} \mid \theta \in \Theta\right\}$.

Definition 3.2 The family is called covariant under the representation $\left\{V_{g}\right\}$ of group $G$ acting on $\Theta$, if

$$
S_{g \theta}=V_{g} S_{\theta} V_{g}^{*}, g \in G, \theta \in \Theta
$$

Assuming that the object is prepared in one of the states $\left\{S_{\theta}\right\}$ but the actual value of $\theta$ is unknown, then the problem is to estimate this value as close as possible from a measurement on the object. We shall treat this problem by methods of the quantum statistical decision theory.

Let $W(\theta, \hat{\theta})$ be a measure of deviation of the measured value $\hat{\theta}$ from the actual value $\theta$. It is natural to assume that $W(\theta, \hat{\theta})$ is invariant:

$$
W(\theta, \hat{\theta})=W(g \theta, g \hat{\theta}) \text { for } g \in G, \theta, \hat{\theta} \in \Theta .
$$

The mean error of the measurement $\Pi \in \mathcal{M}\left(\Theta, \mathcal{H}^{\prime}\right)$ with respect to a deviation measure $W(\theta, \hat{\theta})$, provided that the actual state is $S_{\theta}$, is equal to

$$
\mathcal{D}_{\theta}^{W, S}(\Pi):=\int_{\Theta} W(\theta, \hat{\theta}) \operatorname{tr}\left(\Pi(d \hat{\theta}) S_{\theta}\right)
$$

Following the classical statistical decision theory, we can form two functionals of $\mathcal{D}_{\theta}^{W}$ giving a total measure of precision of the measurement $\Pi$.

In Bayes' approach we take the mean of $\mathcal{D}_{\theta}^{W}$ with respect to a given prior distribution $\pi(d \theta)$. The measurement minimizing the resulting functional:

$$
\mathcal{D}_{\pi}^{W, S}(\Pi):=\int_{\Theta} \mathcal{D}_{\theta}^{W, S}(\Pi) \pi(d \theta)
$$

is called Bayesian. This quantity represents the mean error in the situation where $\theta$ is a random parameter with known distribution $\pi(d \theta)$. In particular, as $\Theta, G$ are compact and "nothing is known" about $\theta$, it is natural to take for $\pi(d \theta)$ the "uniform" distribution, i.e. normalized invariant measure $\nu(d \theta)$ defined as follows:

$$
\nu(B):=\mu(\{g \theta \in B\}) .
$$

It is independent of the choice of $\theta \in \Theta$. This measure $\nu$ is equivalent to the measure defined by the volume bundle induced by Fubini-Study metric.

In minimax approach the maximum possible error with respect to a deviation measure $W(\theta, \hat{\theta})$

$$
\mathcal{D}^{W, S}(\Pi):=\max _{\theta \in \Theta} \mathcal{D}_{\theta}^{W, S}(\Pi)
$$


is minimized. The minimizing measurement is called minimax.

Because $G$ is compact, we shall show that in the covariant case the minima of Bayes and minimax criteria coincide and are achieved on a covariant measurement. We obtain the following quantum Hunt-Stein theorem [5][13]. It is easy to prove the theorem.

Theorem 3.2 For a covariant measurement $\Pi \in \mathcal{M}(\Theta, V)$, we obtain the following equations:

$$
\mathcal{D}_{\theta}^{W, S}(\Pi)=\mathcal{D}_{\nu}^{W, S}(\Pi)=\mathcal{D}^{W, S}(\Pi) .
$$

For $\Pi \in \mathcal{M}\left(\Theta, \mathcal{H}^{\prime}\right)$, denote

$$
\Pi_{g}(B):=V_{g} \Pi\left(B_{g}\right) V_{g}^{*} \text { for } B \in \mathcal{B}(\Theta) .
$$

Introducing the "averaged" measurement

$$
\bar{\Pi}(B):=\int_{G} \Pi_{g^{-1}}(B) \mu(d g),
$$

we have

$$
\mathcal{D}_{\nu}^{W, S}(\bar{\Pi})=\int_{G} \mathcal{D}_{\nu}^{W, S}\left(\Pi_{g^{-1}}\right) \mu(d g)=\mathcal{D}_{\nu}^{W, S}(\Pi) .
$$

Thus,

$$
\mathcal{D}^{W, S}(\Pi) \geq \mathcal{D}_{\nu}^{W, S}(\Pi)=\mathcal{D}_{\nu}^{W, S}(\bar{\Pi}) .
$$

In this case, minimax approach and Bayes' approach with respect to $\nu(d \theta)$ are equivalent. Therefore we minimize the following:

$$
\mathcal{D}_{\theta}^{W, S} \circ V^{\theta}(P)=k^{\prime} \int_{G} W(\theta, g \theta) \operatorname{tr} S_{\theta} V_{g} P V_{g}^{*} \mu(d g)=\operatorname{tr} \hat{W}(\theta) P,
$$

where

$$
\begin{aligned}
\hat{W}(\theta) & :=k^{\prime} \int_{G} W(\theta, g \theta) V_{g}^{*} S_{\theta} V_{g} \mu(d g) \\
& =k^{\prime} \int_{\Theta} W(\theta, \hat{\theta}) S_{\hat{\theta}} \nu(d \hat{\theta}) .
\end{aligned}
$$

Thus, it is sufficient to consider the following minimization:

$$
\min _{P \in \mathcal{S}(\mathcal{H})} \operatorname{tr} \hat{W}(\theta) P=\min _{P \in \mathcal{P}\left(\mathcal{H}^{\prime}\right)} \operatorname{tr} \hat{W}(\theta) P .
$$

\section{Optimal measurement in pure state $n$-i.i.d. model}

In this section we apply the theory of $\S[$ to the problem $\S[2$.

We let as follows:

$$
\Theta:=\mathcal{P}(\mathcal{H}), \mathcal{H}^{\prime}:=\mathcal{H}_{s}^{(n)}, G:=\mathrm{SU}(k), S_{\rho}:=\rho^{(n)} .
$$

We let the action $\left\{V_{g}\right\}$ of $G=\mathrm{SU}(k)$ to $\mathcal{H}_{s}^{(n)}$ be the tensor representation of the natural representation. In this case, $k^{\prime}={ }_{n} H_{k}={ }_{n+k-1} C_{k-1}$. 
Theorem 4.1 If a deviation measure $W(\rho, \hat{\rho})$ is monotone increasing with respect to Fubini-Study distance $d_{f s}$, then we get

$$
\min _{P_{0} \in \mathcal{P}\left(\mathcal{H}_{s}^{(n)}\right)} \operatorname{tr} \hat{W}(\rho) P_{0}=\operatorname{tr} \hat{W}(\rho) \rho^{(n)} .
$$

For a proof see Appendix A. Thus, $V^{\rho}\left(\rho^{(n)}\right)$ is the optimal measurement with respect to a deviation measure $W(\rho, \hat{\rho})$. The optimal measurement is independent of the choice of $\rho$ and $W$ since $V^{\rho_{0}}\left(\rho_{0}^{(n)}\right)=V^{\rho}\left(\rho^{(n)}\right)$. This measurement is denoted by $\Pi_{n}$. The optimal measurements are described as follows:

$$
\Pi_{n}(d \hat{\rho}):=k^{\prime} \hat{\rho}^{(n)} \nu(d \hat{\rho}) .
$$

Under the following chart (四), the optimal measurements are denoted as:

$$
\Pi_{n}(d \theta)=k^{\prime}\left|\phi(\theta)^{(n)}\right\rangle\left\langle\phi(\theta)^{(n)}\right| \nu(d \theta)
$$

for $\theta \in\left\{\theta \in \mathbf{R}^{2 k-2} \mid \theta_{i} \in[0,2 \pi) 1 \leq i \leq k-1, \theta_{j} \in[0, \pi / 2]\right\}$, where we defined as follows:

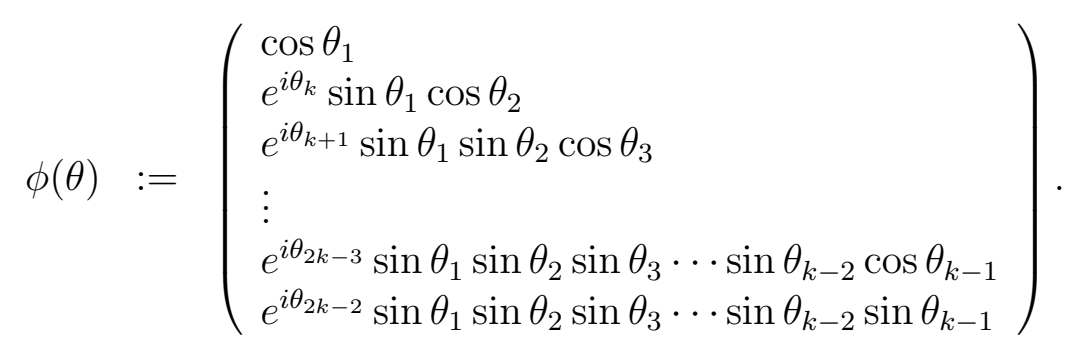

The invariant measures $\nu(d \theta)$ is described in this chart as: (See pp.31 in [14].)

$\nu(d \theta)=\frac{(k-1) !}{\pi^{k-1}} \sin ^{2 k-3} \theta_{1} \sin ^{2 k-5} \theta_{2} \cdots \sin \theta_{k-1} \cos \theta_{1} \cos \theta_{2} \cdots \cos \theta_{k-1} d \theta_{1} d \theta_{2} \cdots d \theta_{2 k-2}$.

Lemma 4.1 If $W(\rho, \hat{\rho})=h \circ d_{f s}(\rho, \hat{\rho})$, then

$$
\mathcal{D}^{W,(n)}\left(\Pi_{n}\right)=2(k-1){ }_{n} H_{k} \int_{0}^{\frac{\pi}{2}} h(\theta) \cos ^{2 n+1} \theta \sin ^{2 k-3} \theta d \theta .
$$

For a proof, see Appendix B.

\section{Deviation measures by distances}

First, we calculate deviation measures by Bures's distance $d_{b}$. (See Definition 2.1.) We obtain that

$$
\mathcal{D}^{d_{b}^{\gamma},(n)}\left(\Pi_{n}\right)=2(k-1){ }_{n} H_{k} \int_{0}^{\frac{\pi}{2}} \cos ^{2 n+1} \theta \sin ^{2 k-3+\gamma} \theta d \theta .
$$


Theorem 5.1 We obtain the following equation.

$$
\lim _{n \rightarrow \infty} \mathcal{D}^{d_{b}^{\gamma},(n)}\left(\Pi_{n}\right) n^{\frac{\gamma}{2}}=\frac{\Gamma(k-1+\gamma / 2)}{\Gamma(k-1)} .
$$

Specially in the case of $\gamma=2$, we have

$$
\mathcal{D}^{d_{b}^{2},(n)}\left(\Pi_{n}\right) n=\frac{(k-1) n}{n+k} \rightarrow k-1 .
$$

Proof Because of Lemma 5.2,

$$
\begin{aligned}
\mathcal{D}^{d_{b}^{\gamma},(n)}\left(\Pi_{n}\right) n^{\frac{\gamma}{2}} & =2(k-1){ }_{n} H_{k} \frac{n^{\gamma / 2} \Gamma(n+1) \Gamma(k-1+\gamma / 2)}{\Gamma(n+k+\gamma / 2)} \\
& =\frac{n^{\gamma / 2} \Gamma(n+1) \Gamma(k-1+\gamma / 2) \Gamma(n+k)}{\Gamma(n+k+\gamma / 2) \Gamma(n+1) \Gamma(k-1)} \\
& =\frac{\Gamma(n+k) n^{\gamma / 2}}{\Gamma(n+k+\gamma / 2)} \frac{\Gamma(k-1+\gamma / 2)}{\Gamma(k-1)} \\
& \rightarrow \frac{\Gamma(k-1+\gamma / 2)}{\Gamma(k-1)} \text { as } n \rightarrow \infty,
\end{aligned}
$$

where the limit is induced by the following formula of $\Gamma$ function:

$$
\lim _{n \rightarrow \infty} \frac{\Gamma(n+x)}{\Gamma(n) n^{x}}=1 .
$$

Letting $\gamma:=2$, we obtain

$$
\begin{aligned}
\mathcal{D}^{d_{b}^{2},(n)}\left(\Pi_{n}\right) & =\frac{\Gamma(n+k)}{\Gamma(n+k+1)} \frac{\Gamma(k-1+1)}{\Gamma(k-1)} \\
& =\frac{k-1}{n+k} .
\end{aligned}
$$

Next, we consider the deviation measure by Fubini-Study distance. (See Definition 2.1.)

Theorem 5.2 We obtain the following equation:

$$
\lim _{n \rightarrow \infty} \mathcal{D}_{f s}^{d_{f}^{2}(n)}\left(\Pi_{n}\right) n=k-1 .
$$

Proof Because

$$
\mathcal{D}^{d_{f s}^{2},(n)}\left(\Pi_{n}\right)=2(k-1){ }_{n} H_{k} \int_{0}^{\frac{\pi}{2}} \theta^{2} \cos ^{2 n+1} \theta \sin ^{2 k-3} \theta d \theta,
$$

from Theorem 5.1, it is sufficient to prove that

$$
\frac{\int_{0}^{\frac{\pi}{2}} \cos ^{2 n+1} \theta \sin ^{2 k-1} \theta d \theta}{\int_{0}^{\frac{\pi}{2}} \theta^{2} \cos ^{2 n+1} \theta \sin ^{2 k-3} \theta d \theta} \rightarrow 1 \text { as } n \rightarrow \infty .
$$

From Lemma 5.1, the proof is complete. 
Lemma 5.1 Let $f_{n}, g_{n}$ be continuous nonnegative functions on $[0, a]$. If $f_{n}, g_{n}$ satisfies the following two conditions:

$$
\begin{aligned}
& \text { (1) } \frac{\int_{\delta}^{a} f_{n}(x) d x}{\int_{0}^{a} f_{n}(x) d x}, \frac{\int_{\delta}^{a} g_{n}(x) d x}{\int_{0}^{a} g_{n}(x) d x} \rightarrow 0 \text { as } n \rightarrow \infty \text { for } \forall \delta \in(0, a) \\
& \text { (2) } \frac{f_{n}(x)}{g_{n}(x)} \rightarrow 1 \text { as } x \rightarrow 0 \text { uniformally w.r.t. } n
\end{aligned}
$$

then we have

$$
\frac{\int_{0}^{a} f_{n}(x) d x}{\int_{0}^{a} g_{n}(x) d x} \rightarrow 1 \text { as } n \rightarrow \infty
$$

For a proof, see Appendix Q.

Lemma 5.2 For any $x, y$, we have

$$
\int_{0}^{\frac{\pi}{2}} \cos ^{x} \theta \sin ^{y} \theta d \theta=\frac{\Gamma\left(\frac{x+1}{2}\right) \Gamma\left(\frac{y+1}{2}\right)}{2 \Gamma\left(\frac{x+y}{2}+1\right)} .
$$

Specially, in the case of $x=2 k+1, y=2 l+1$.

$$
\int_{0}^{\frac{\pi}{2}} \cos ^{2 k+1} \theta \sin ^{2 l+1} \theta d \theta=\frac{k ! l !}{2(k+l+1) !} .
$$

Proof Letting $t:=\cos ^{2} \theta$, we get

$$
\begin{aligned}
\int_{0}^{\frac{\pi}{2}} \cos ^{x} \theta \sin ^{y} \theta d \theta & =\frac{1}{2} \int_{0}^{1} t^{\frac{x-1}{2}}(1-t)^{\frac{y-1}{2}} d t \\
& =\frac{1}{2} B\left(\frac{x+1}{2}, \frac{y+1}{2}\right) \\
& =\frac{\Gamma\left(\frac{x+1}{2}\right) \Gamma\left(\frac{y+1}{2}\right)}{2 \Gamma\left(\frac{x+y}{2}+1\right)} .
\end{aligned}
$$

where we use the following formula of $B$ function:

$$
B(x, y)=\int_{0}^{1} t^{x-1}(1-t)^{y-1} d t=\frac{\Gamma(x) \Gamma(y)}{\Gamma(x+y)} .
$$

\section{Global large deviation evaluation}

In this section, we will estimate the global large deviation evaluation by the optimal measurement given in $\S$. 
Theorem 6.1 We obtain the following:

$$
\begin{aligned}
& \min _{M_{n} \in \mathcal{M}\left(\mathcal{P}(\mathcal{H}), \mathcal{H}_{s}^{(n)}\right)} \lim _{n \rightarrow \infty} \max _{\rho \in \mathcal{P}(\mathcal{H})} \frac{1}{n} \log \operatorname{Pr}_{M_{n}}^{\rho^{(n)}}\left\{\hat{\rho} \in \mathcal{P}(\mathcal{H}) \mid d_{f s}(\rho, \hat{\rho}) \geq \epsilon\right\} \\
& =\lim _{n \rightarrow \infty} \min _{M_{n} \in \mathcal{M}\left(\mathcal{P}(\mathcal{H}), \mathcal{H}_{s}^{(n)}\right)} \max _{\rho \in \mathcal{P}(\mathcal{H})} \frac{1}{n} \log \operatorname{Pr}_{M_{n}}^{\rho^{(n)}}\left\{\hat{\rho} \in \mathcal{P}(\mathcal{H}) \mid d_{f s}(\rho, \hat{\rho}) \geq \epsilon\right\} \\
& =\lim _{n \rightarrow \infty} \frac{1}{n} \log \operatorname{Pr}_{\Pi_{n}}^{\rho^{(n)}}\left\{\hat{\rho} \in \mathcal{P}(\mathcal{H}) \mid d_{f s}(\rho, \hat{\rho}) \geq \epsilon\right\} \\
& =2 \log \cos \epsilon \\
& \lim _{\epsilon \rightarrow 0} \lim _{n \rightarrow \infty} \min _{M_{n} \in \mathcal{M}\left(\mathcal{P}(\mathcal{H}), \mathcal{H}_{s}^{(n)}\right)} \max _{\rho \in \mathcal{P}(\mathcal{H})} \frac{1}{\epsilon^{2} n} \log \operatorname{Pr}_{M_{n}}^{\rho^{(n)}}\left\{\hat{\rho} \in \mathcal{P}(\mathcal{H}) \mid d_{f s}(\rho, \hat{\rho}) \geq \epsilon\right\} \\
& =\min _{M_{n} \in \mathcal{M}\left(\mathcal{P}(\mathcal{H}), \mathcal{H}_{s}^{(n)}\right)} \lim _{\epsilon \rightarrow 0} \lim _{n \rightarrow \infty} \max _{\rho \in \mathcal{P}(\mathcal{H})} \frac{1}{\epsilon^{2} n} \log \operatorname{Pr}_{M_{n}}^{\rho^{(n)}}\left\{\hat{\rho} \in \mathcal{P}(\mathcal{H}) \mid d_{f s}(\rho, \hat{\rho}) \geq \epsilon\right\} \\
& =\lim _{\epsilon \rightarrow 0} \lim _{n \rightarrow \infty} \frac{1}{\epsilon^{2} n} \log \operatorname{Pr}_{\Pi_{n}}^{\rho^{(n)}}\left\{\hat{\rho} \in \mathcal{P}(\mathcal{H}) \mid d_{f s}(\rho, \hat{\rho}) \geq \epsilon\right\} \\
& =-1 \text {, }
\end{aligned}
$$

where $\operatorname{Pr}_{M}^{S} B$ denotes the probability of $B$ with respect to the probability measure $\operatorname{tr}(M(d \omega) S)$ for a Borel $B \subset \Omega$, a measurement $M \in \mathcal{M}\left(\Omega, \mathcal{H}^{\prime}\right)$, and a state $S \in \mathcal{S}\left(\mathcal{H}^{\prime}\right)$.

Proof Because

$$
\frac{\log { }_{n} H_{k}}{n} \leq \frac{\log (n+1)^{k}}{n}=\frac{k \log (n+1)}{n} \rightarrow 0
$$

then

$$
\begin{aligned}
\lim _{n \rightarrow \infty} \frac{1}{n} \log \operatorname{Pr}_{\Pi_{n}}^{\rho^{(n)}}\left\{\hat{\rho} \in \mathcal{P}(\mathcal{H}) \mid d_{f s}(\rho, \hat{\rho}) \geq \epsilon\right\} & =\lim _{n \rightarrow \infty} \frac{1}{n} \log \left(2(k-1){ }_{n} H_{k} \int_{\epsilon}^{\frac{\pi}{2}} \cos ^{2 n+1} \theta \sin ^{2 k-3} \theta d \theta\right) \\
& =\lim _{n \rightarrow \infty} \frac{1}{n} \log \int_{\epsilon}^{\frac{\pi}{2}} \cos ^{2 n+1} \theta \sin ^{2 k-3} \theta d \theta .
\end{aligned}
$$

Letting $x:=\cos \theta$, we have

$$
\int_{\epsilon}^{\frac{\pi}{2}} \cos ^{2 n+1} \theta \sin ^{2 k-3} \theta d \theta=\int_{0}^{\cos \epsilon} x^{2 n+1}\left(1-x^{2}\right)^{k-2} d x .
$$

The preceding equation is evaluate as follows:

$$
\int_{0}^{\cos \epsilon} x^{2 n+1}\left(1-x^{2}\right)^{k-2} d x \leq \cos ^{2 n+1} \epsilon \int_{0}^{\cos \epsilon}\left(1-x^{2}\right)^{k-2} d x \leq \cos ^{2 n+2} \epsilon .
$$

The preceding equation is evaluate as follows:

$$
\int_{0}^{\cos \epsilon} x^{2 n+1}\left(1-x^{2}\right)^{k-2} d x \geq\left(1-\cos ^{2} \epsilon\right)^{k-2} \int_{0}^{\cos \epsilon} x^{2 n+1} d x=\frac{\left(1-\cos ^{2} \epsilon\right)^{k-2}}{2 n+2} \cos ^{2(n+1)} \epsilon .
$$


Therefore, $\frac{1}{n} \log \int_{0}^{\cos \epsilon} x^{2 n+1}\left(1-x^{2}\right)^{k-2} d x \rightarrow \log \cos ^{2} \epsilon$ because $\frac{1}{n} \log \frac{\left(1-\cos ^{2} \epsilon\right)^{k-2}}{2 n+2} \rightarrow 0$. We have

$$
\lim _{n \rightarrow \infty} \frac{1}{n} \log \operatorname{Pr}_{\Pi_{n}}^{\rho^{(n)}}\left\{\hat{\rho} \in \mathcal{P}(\mathcal{H}) \mid d_{f s}(\rho, \hat{\rho}) \geq \epsilon\right\}=2 \log \cos \epsilon
$$

We obtain (6). Since

$$
\lim _{\epsilon \rightarrow 0} \frac{2}{\epsilon^{2}} \log \cos \epsilon=-1
$$

we obtain $(7)$.

\section{$7 \quad$ Fisher information on $\operatorname{tr} \Pi_{n}(d \hat{\rho}) \rho^{(n)}$}

In this section, we calculate the Fisher information of $n$ tensor model by standard optimum measurement. $J_{\Pi_{n}}^{\rho}$ denotes the Fisher information. We consider the tangent space $T_{\rho} \mathcal{P}(\mathcal{H})$ at $\rho:=|\phi(0)\rangle\langle\phi(0)|$. If $c(t)$ is a curve on $\mathcal{P}(\mathcal{H})$ such that $c(0)=\rho, \dot{c}$ denotes the element of $T_{\rho} \mathcal{P}(\mathcal{H})$ defined by $c(t)$. Fubini-Study metric $g_{f s}$ is defined as:

$$
g_{f s}(\dot{c}, \dot{c}):=\left(\lim _{t \rightarrow 0} \frac{d_{f s}(c(0), c(t))}{t}\right)^{2}
$$

Theorem 7.1 We have

$$
J_{\Pi_{n}}^{\rho}=2 n g_{f s}
$$

Since $d_{J_{\Pi_{1}}^{\rho}}=\sqrt{2} d_{f s}$, from Lemma 7.1, we obtain the following Corollary, where $d_{g}$ denotes the geodesic distance with respect to a Riemannian metric $g$.

Corollary 7.1 Let a measurement $T_{(n)}$ be the maximum likelihood estimation of $n$ data given by the measurement $\underbrace{\Pi_{1} \otimes \Pi_{1} \otimes \cdots \otimes \Pi_{1}}_{n}$. We obtain the following.

$$
\lim _{\epsilon \rightarrow 0} \lim _{n \rightarrow \infty} \frac{1}{\epsilon^{2} n} \log \operatorname{Pr}_{T_{(n)}^{\rho^{(n)}}}\left\{\hat{\rho} \in \mathcal{P}(\mathcal{H}) \mid d_{f s}(\rho, \hat{\rho}) \geq \epsilon\right\}=-1
$$

Lemma 7.1 Let $\left\{p_{\theta} \mid \theta \in \Theta\right\}$ be a family of probability distributions. If $T^{(n)}$ is a consistent estimator, then we have

$$
\lim _{\epsilon \rightarrow 0} \lim _{n \rightarrow \infty} \frac{1}{\epsilon^{2} n} \log p_{\theta}\left\{d_{J}\left(T^{(n)}, \theta\right) \geq \epsilon\right\} \geq-\frac{1}{2},
$$

where $J$ denotes the Fisher metric of $\left\{p_{\theta} \mid \theta \in \Theta\right\}$. The equality establishes if $T^{(n)}$ is the maximum likelihood estimation. 
Proof of Theorem 7.1 Let $c(t):=\left|\phi_{t}\right\rangle\left\langle\phi_{t}\right|, \phi_{t}:=\phi(t, 0, \ldots, 0)$. (See the equation (4).) Because $g_{f s}(\dot{c}, \dot{c})=1$, it is sufficient to prove that

$$
J_{\Pi_{n}}^{\rho}(\dot{c}, \dot{c})=2 n .
$$

We have

$$
\begin{aligned}
& \left|\left\langle\phi_{t} \mid \phi(\theta)\right\rangle\right|^{2 n} \\
= & \left(\cos ^{2} t \cos ^{2} \theta_{1}+\sin ^{2} \theta_{1} \cos ^{2} \theta_{2} \sin ^{2} t+2 \cos t \sin t \cos \theta_{1} \sin \theta_{1} \cos \theta_{2} \cos \theta_{k}\right)^{n} \\
& \left(\left.\frac{d}{d t} \log \left(\left|\left\langle\phi_{t} \mid \phi(\theta)\right\rangle\right|^{2 n}\right)\right|_{t=0}\right)^{2}\left|\left\langle\phi_{0} \mid \phi(\theta)\right\rangle\right|^{2 n} \\
= & \left(2 n \cos \theta_{1} \sin \theta_{1} \cos \theta_{2} \cos \theta_{k}\right)^{2} \cos ^{2 n-4} \theta_{1} \\
= & 4 n^{2} \cos ^{2 n-2} \theta_{1} \sin ^{2} \theta_{1} \cos ^{2} \theta_{2} \cos ^{2} \theta_{k} \\
& \operatorname{tr} \Pi_{n}(d \theta) \rho^{(n)} \\
= & { }_{n} H_{k} \frac{(k-1) !}{\pi^{k-1}} \frac{(2 \pi)^{k-2}}{2^{k-3}(k-3) !} \int_{\mathcal{P}(\mathcal{H})}\left|\left\langle\phi_{t} \mid \phi(\theta)\right\rangle\right|^{2 n} \nu(d \theta) \\
= & \frac{2(k-1)(k-2)}{\pi}{ }_{n} H_{k} \int_{0}^{2 \pi} \int_{0}^{\frac{\pi}{2}} \int_{0}^{\frac{\pi}{2}}\left|\left\langle\phi_{t} \mid \phi(\theta)\right\rangle\right|^{2 n} \sin ^{2 k-3} \theta_{1} \cos \theta_{1} d \theta_{1} \sin ^{2 k-5} \theta_{2} \cos \theta_{2} d \theta_{2} d \theta_{k} .
\end{aligned}
$$

Therefore, we obtain

$$
\begin{aligned}
& J_{\Pi_{n}}^{\rho}(\dot{c}, \dot{c}) \\
= & \frac{2(k-1)(k-2)}{\pi}{ }_{n} H_{k} \times \\
& \int_{0}^{2 \pi} \int_{0}^{\frac{\pi}{2}} \int_{0}^{\frac{\pi}{2}}\left(\left.\frac{d}{d t} \log \left(\left|\left\langle\phi_{t} \mid \phi(\theta)\right\rangle\right|^{2 n}\right)\right|_{t=0}\right)^{2}\left|\left\langle\phi_{0} \mid \phi(\theta)\right\rangle\right|^{2 n} \sin ^{2 k-3} \theta_{1} \cos \theta_{1} d \theta_{1} \sin ^{2 k-5} \theta_{2} \cos \theta_{2} d \theta_{2} d \theta_{k} \\
= & \frac{2(k-1)(k-2)}{\pi}{ }_{n} H_{k} 4 n^{2} \int_{0}^{\frac{\pi}{2}} \cos ^{2 n-1} \theta_{1} \sin ^{2 k-1} \theta_{1} d \theta_{1} \cdot \int_{0}^{\frac{\pi}{2}} \cos ^{3} \theta_{2} \sin ^{2 k-5} \theta_{2} d \theta_{2} \int_{0}^{2 \pi} \cos ^{2} \theta_{k} d \theta_{k} \\
= & \frac{2(k-1)(k-2)}{\pi}{ }_{n} H_{k} 4 n^{2} \frac{(n-1) !(k-1) !}{2(n+k-1) !} \frac{1 !(k-3) !}{2(k-1) !} \pi \\
= & 2 n .
\end{aligned}
$$

\section{Conclusion}

In this paper, we have that the effect of the quantum i.i.d. expansion vanishes in the sense of large deviation. Since the family $\left\{\operatorname{tr} \Pi_{1}(d \hat{\rho}) \rho \mid \rho \in \mathcal{P}(\mathcal{H})\right\}$ of probability distributions is symmetric under the action of $\mathrm{SU}(k)$, the process needed by the classical maximum likelihood estimation on $n$ data is easier than the construction of the optimal measurement on $n$-i.i.d. model. This result depends on the effect of a pure state. If we consider the model which consists of mixed states, we have to note the gap between the classical statistical estimation based on $n$ data of the optimal measurement on 1-particle system and the optimal measurement on $n$-particle system. 


\section{Appendices}

\section{A Proof of Theorem 4.1}

In this Appendix, assume that $\rho=|\phi(0)\rangle\langle\phi(0)|$. Because $\mathcal{H}_{s}^{(n)}$ is irreducible with respect to the action of $\mathrm{SU}(k)$,

$$
\begin{aligned}
\mathcal{H}_{s}^{(n)} & =\left\{\sum_{i} a_{i} V_{g_{i}} \phi(0)^{(n)} \mid a_{i} \in \mathbf{C}, g_{i} \in \mathrm{SU}(k)\right\} \\
& =\left\{\sum_{i} \phi_{i}^{(n)} \mid \phi \in \mathcal{H}\right\} .
\end{aligned}
$$

We assume that $W(\rho, \hat{\rho})=h(\operatorname{tr} \rho \hat{\rho})$. As $h$ is monotone decreasing, there exists a measure $h^{\prime}$ on $[0,1]$ such that $h(x)=h^{\prime}([x, 1])$.

The function $h_{\beta}$ on $[0,1]$ and the deviation measure $W_{\beta}$ are defined as follows:

$$
\begin{aligned}
h_{\beta}(x) & :=\left\{\begin{array}{lll}
1 & \text { for } & x \leq \beta \\
0 & \text { for } & x>\beta
\end{array}\right. \\
W_{\beta}(\rho, \hat{\rho}) & :=h_{\beta}(\operatorname{tr} \rho \hat{\rho}) .
\end{aligned}
$$

From Lemma A.1, for any measurement $\Pi$ we have

$$
\mathcal{D}_{\rho}^{W,(n)}(\Pi)=\int_{[0,1]} \mathcal{D}_{\rho}^{W_{\beta},(n)}(\Pi) h^{\prime}(d \beta) .
$$

From (9), it is sufficient to show the following for $\left\{\phi_{i}\right\} \subset \mathcal{H}$ in the case of $W=W_{\beta}$.

$$
\frac{\operatorname{tr} \hat{W}_{\beta}(\rho)\left|\sum_{i} \phi_{i}^{(n)}\right\rangle\left\langle\sum_{i} \phi_{i}^{(n)}\right|}{\left\langle\sum_{i} \phi_{i}^{(n)} \mid \sum_{i} \phi_{i}^{(n)}\right\rangle} \geq \operatorname{tr} \hat{W}_{\beta}(\rho)\left|\phi(0)^{(n)}\right\rangle\left\langle\phi(0)^{(n)}\right| .
$$

From Lemma A.2 it is sufficient for (10) to prove the following:

$$
\begin{aligned}
& \left\langle\sum_{i} \phi_{i}^{(n)}\left|\hat{W}_{\beta}(\rho)\right| \sum_{i} \phi_{i}^{(n)}\right\rangle \cdot\left\langle\phi(0)^{(n)}\left|\operatorname{Id}-\hat{W}_{\beta}(\rho)\right| \phi(0)^{(n)}\right\rangle \\
\geq & \left\langle\phi(0)^{(n)}\left|\hat{W}_{\beta}(\rho)\right| \phi(0)^{(n)}\right\rangle \cdot\left\langle\sum_{i} \phi_{i}^{(n)}\left|\operatorname{Id}-\hat{W}_{\beta}(\rho)\right| \sum_{i} \phi_{i}^{(n)}\right\rangle .
\end{aligned}
$$

Remark that $|\langle\phi(\theta) \mid \phi(0)\rangle|^{2}=\cos ^{2} \theta_{1}$. From Lemma A.3, we get

$$
\begin{aligned}
\left\langle\sum_{i} \phi_{i}^{(n)}\left|\hat{W}_{\beta}(\rho)\right| \sum_{i} \phi_{i}^{(n)}\right\rangle & =\frac{k^{\prime} \cdot(k-1) !}{\pi^{(k-1)}} \int_{\alpha}^{\frac{\pi}{2}} f_{1}\left(\theta_{1}\right) \cos \theta_{1} \sin ^{2 k-3} \theta_{1} d \theta_{1} \\
\left\langle\sum_{i} \phi_{i}^{(n)}\left|\operatorname{Id}-\hat{W}_{\beta}(\rho)\right| \sum_{i} \phi_{i}^{(n)}\right\rangle & =\frac{k^{\prime} \cdot(k-1) !}{\pi^{(k-1)}} \int_{0}^{\alpha} f_{1}\left(\theta_{1}\right) \cos \theta_{1} \sin ^{2 k-3} \theta_{1} d \theta_{1} \\
\left\langle\phi(0)^{(n)}\left|\hat{W}_{\beta}(\rho)\right| \phi(0)^{(n)}\right\rangle & =C \int_{\alpha}^{\frac{\pi}{2}} \cos ^{2 n+1} \theta_{1} \sin ^{2 k-3} \theta_{1} d \theta_{1} \\
\left\langle\phi(0)^{(n)}\left|\operatorname{Id}-\hat{W}_{\beta}(\rho)\right| \phi(0)^{(n)}\right\rangle & =C \int_{0}^{\alpha} \cos ^{2 n+1} \theta_{1} \sin ^{2 k-3} \theta_{1} d \theta_{1},
\end{aligned}
$$


where

$$
\begin{aligned}
\beta & :=\cos ^{2} \alpha \\
f_{1}\left(\theta_{1}\right) & :=\underbrace{\int_{0}^{\frac{\pi}{2}} \cdots \int_{0}^{\frac{\pi}{2}}}_{k-2} f_{2}\left(\theta_{1}, \ldots, \theta_{k-1}\right) \lambda\left(d \theta_{2} \cdots d \theta_{k-1}\right) \\
f_{2}\left(\theta_{1}, \ldots, \theta_{k-1}\right) & :=\underbrace{\int_{0}^{2 \pi} \cdots \int_{0}^{2 \pi} \sum_{i, j}\left\langle\phi_{i} \mid \phi(\theta)\right\rangle^{n}\left\langle\phi(\theta) \mid \phi_{j}\right\rangle^{n} d \theta_{k} \cdots d \theta_{2 k-2}}_{k-1} \\
C & :=\underbrace{k^{\prime} \cdot(k-1) !}_{\pi^{(k-1)}} \underbrace{\frac{\pi}{2}}_{0} \ldots \int_{0}^{\frac{\pi}{2}} \underbrace{\int_{0}^{2 \pi} \cdots \int_{0}^{2 \pi}}_{k-1} \lambda\left(d \theta_{2} d \theta_{3} \cdots d \theta_{k-1}\right) d \theta_{k} \cdots d \theta_{2 k-2} \\
\lambda\left(d \theta_{2}, \ldots, d \theta_{k-1}\right) & :=\sin ^{2 k-5} \theta_{2} \cdots \sin \theta_{k-1} \cos \theta_{2} \cdots \cos \theta_{k-1} d \theta_{2} \cdots d \theta_{k-1} .
\end{aligned}
$$

Therefore, it is sufficient for the equation (11) to show that for $\pi / 2 \geq \theta_{1}>\theta_{1}^{\prime} \geq 0$

$$
f_{1}\left(\theta_{1}\right) \sin ^{2 k-3} \theta_{1} \cos ^{2 n+1} \theta_{1}^{\prime} \sin ^{2 k-3} \theta_{1}^{\prime} \geq f_{1}\left(\theta_{1}^{\prime}\right) \sin ^{2 k-3} \theta_{1}^{\prime} \cos ^{2 n+1} \theta_{1} \sin ^{2 k-3} \theta_{1} .
$$

It suffices to verify that for $\theta_{i} \in\left[0, \frac{\pi}{2}\right], 2 \leq i \leq k-1, \pi / 2 \geq \theta_{1}>\theta_{1}^{\prime} \geq b$

$$
\frac{f_{2}\left(\theta_{1}, \theta_{2}, \ldots, \theta_{n-1}\right)}{\cos ^{2 n} \theta_{1}} \geq \frac{f_{2}\left(\theta_{1}^{\prime}, \theta_{2}, \ldots, \theta_{n-1}\right)}{\cos ^{2 n} \theta_{1}^{\prime}}
$$

Thus, it is sufficient to prove that the following is monotone decreasing about $\theta_{1}$ for any $\theta_{2}, \ldots, \theta_{k-1}$ :

$$
\frac{1}{\cos ^{2 n} \theta_{1}} \underbrace{\int_{0}^{2 \pi} \cdots \int_{0}^{2 \pi}}_{k-1} \sum_{i, j}\left\langle\phi_{i} \mid \phi(\theta)\right\rangle^{n}\left\langle\phi(\theta) \mid \phi_{j}\right\rangle^{n} d \theta_{k} \cdots d \theta_{2 k-2} .
$$

Letting

$$
\phi_{i}:=\left(\begin{array}{c}
e^{i \psi_{i}^{1}} \phi_{i}^{1} \\
e^{i \psi_{i}^{2}} \phi_{i}^{2} \\
\vdots \\
e^{i \psi_{i}^{k}} \phi_{i}^{k}
\end{array}\right)
$$

we get

$$
\begin{aligned}
& \frac{\left\langle\phi_{i} \mid \phi(\theta)\right\rangle^{n}}{\cos ^{n} \theta_{1}} \\
= & \left(e^{i \psi_{i}^{1}} \phi_{i}^{1}+\sum_{j=2}^{k-1} e^{i\left(\theta_{k-2+j}-\psi_{i}^{j}\right)} \tan \theta_{1} \sin \theta_{2} \cdots \sin \theta_{j} \cos \theta_{j+1} \phi_{i}^{j}\right. \\
& \left.+e^{i\left(\theta_{2 k-2}-\psi_{i}^{k-1}\right)} \tan \theta_{1} \sin \theta_{2} \cdots \sin \theta_{k-1} \phi_{i}^{k}\right)^{n} .
\end{aligned}
$$

Letting $x:=\tan \theta_{1}$, Lemma A.4 induce that (12) is monotone decreasing about $\theta_{1}$. The proof is complete. 
Lemma A.1 If the deviation measure $W(\rho, \hat{\rho})=h^{\prime}([\operatorname{tr} \rho \hat{\rho}, 1])$, then

$$
\mathcal{D}_{\rho}^{W,(n)}(\Pi)=\int_{[0,1]} \mathcal{D}_{\rho}^{W_{\beta},(n)}(\Pi) h^{\prime}(d \beta) .
$$

Proof For the probability measure $\pi$ on $\mathcal{P}(\mathcal{H})$, we have

$$
\begin{aligned}
\int_{\mathcal{P}(\mathcal{H})} W(\rho, \hat{\rho}) \pi(d \hat{\rho}) & =\int_{\mathcal{P}(\mathcal{H})} h(\operatorname{tr} \rho \hat{\rho}) \pi(d \hat{\rho}) \\
& =\int_{\mathcal{P}(\mathcal{H})} \int_{[0,1]} h_{\beta}(\operatorname{tr} \rho \hat{\rho}) h^{\prime}(d \beta) \pi(d \hat{\rho}) \\
& =\int_{[0,1]}\left(\int_{\mathcal{P}(\mathcal{H})} h_{\beta}(\operatorname{tr} \rho \hat{\rho}) \pi(d \hat{\rho})\right) h^{\prime}(d \beta) \\
& =\int_{[0,1]}\left(\int_{\mathcal{P}(\mathcal{H})} W_{\beta}(\rho, \hat{\rho}) \pi(d \hat{\rho})\right) h^{\prime}(d \beta) .
\end{aligned}
$$

Substituting $\pi(d \hat{\rho})$ for $\operatorname{tr}\left(\Pi(d \hat{\rho}) \rho^{(n)}\right)$, then we obtain (13).

Lemma A.2 Let $\mathcal{H}$ be any finite dimensional Hilbert space. For any elements $\phi, \psi \in \mathcal{H}$ and any selfadjoint operator $A$ on $\mathcal{H}$, the following are equivalent.

$$
\begin{aligned}
& \frac{\langle\phi|A| \phi\rangle}{\langle\phi \mid \phi\rangle} \geq \frac{\langle\psi|A| \psi\rangle}{\langle\psi \mid \psi\rangle} . \\
\circ & \langle\phi|A| \phi\rangle\langle\psi|\operatorname{Id}-A| \psi\rangle \geq\langle\psi|A| \psi\rangle\langle\phi|\operatorname{Id}-A| \phi\rangle .
\end{aligned}
$$

Lemma A.3 we have

$$
\begin{aligned}
\left\langle\sum_{i} \phi_{i}^{(n)}\left|\hat{W}_{\beta}(\rho)\right| \sum_{i} \phi_{i}^{(n)}\right\rangle & =\frac{k^{\prime} \cdot(k-1) !}{\pi^{(k-1)}} \int_{\alpha}^{\frac{\pi}{2}} f_{1}\left(\theta_{1}\right) \cos \theta_{1} \sin ^{2 k-3} \theta_{1} d \theta_{1} \\
\left\langle\sum_{i} \phi_{i}^{(n)}\left|\operatorname{Id}-\hat{W}_{\beta}(\rho)\right| \sum_{i} \phi_{i}^{(n)}\right\rangle & =\frac{k^{\prime} \cdot(k-1) !}{\pi^{(k-1)}} \int_{0}^{\alpha} f_{1}\left(\theta_{1}\right) \cos \theta_{1} \sin ^{2 k-3} \theta_{1} d \theta_{1} \\
\left\langle\phi(0)^{(n)}\left|\hat{W}_{\beta}(\rho)\right| \phi(0)^{(n)}\right\rangle & =C \int_{\alpha}^{\frac{\pi}{2}} \cos ^{2 n+1} \theta_{1} \sin ^{2 k-3} \theta_{1} d \theta_{1} \\
\left\langle\phi(0)^{(n)}\left|\operatorname{Id}-\hat{W}_{\beta}(\rho)\right| \phi(0)^{(n)}\right\rangle & =C \int_{0}^{\alpha} \cos ^{2 n+1} \theta_{1} \sin ^{2 k-3} \theta_{1} d \theta_{1} .
\end{aligned}
$$

Proof $\hat{W}_{\beta}(\rho)$ is denoted as follows:

$$
\begin{aligned}
\hat{W}_{\beta}(\rho) & =k^{\prime} \int_{\mathcal{P}(\mathcal{H})} W_{\beta}(\rho, \hat{\rho}) \hat{\rho}^{(n)} \nu(d \hat{\rho}) \\
& =k^{\prime} \int_{\{\hat{\rho} \in \mathcal{P}(\mathcal{H}) \mid \operatorname{tr} \hat{\rho} \rho \leq \beta\}} \hat{\rho}^{(n)} \nu(d \hat{\rho}) .
\end{aligned}
$$

We obtain

$$
\begin{aligned}
\left\langle\sum_{i} \phi_{i}^{(n)}\left|\hat{W}_{\beta}(\rho)\right| \sum_{i} \phi_{i}^{(n)}\right\rangle & =\left\langle\sum_{i} \phi_{i}^{(n)}\left|k^{\prime} \int_{\{\hat{\rho} \in \mathcal{P}(\mathcal{H}) \mid \operatorname{tr} \hat{\rho} \rho \leq \beta\}} \hat{\rho}^{(n)} \nu(d \hat{\rho})\right| \sum_{i} \phi_{i}^{(n)}\right\rangle \\
& =\sum_{i, j} k^{\prime} \int_{\{\hat{\rho} \in \mathcal{P}(\mathcal{H}) \mid \operatorname{tr} \hat{\rho} \rho \leq \beta\}}\left\langle\phi_{i}^{(n)}\left|\hat{\rho}^{(n)}\right| \phi_{j}^{(n)}\right\rangle \nu(d \hat{\rho}) \\
& =\sum_{i, j} k^{\prime} \int_{\{\hat{\rho} \in \mathcal{P}(\mathcal{H}) \mid \operatorname{tr} \hat{\rho} \rho \leq \beta\}}\left\langle\phi_{i}|\hat{\rho}| \phi_{j}\right\rangle^{n} \nu(d \hat{\rho}) \\
& =\frac{k^{\prime} \cdot(k-1) !}{\pi^{(k-1)}} \int_{\alpha}^{\frac{\pi}{2}} f_{1}\left(\theta_{1}\right) \cos \theta_{1} \sin ^{2 k-3} \theta_{1} d \theta_{1} .
\end{aligned}
$$


Similarly,

$$
\begin{aligned}
\left\langle\sum_{i} \phi_{i}^{(n)}\left|\operatorname{Id}-\hat{W}_{\beta}(\rho)\right| \sum_{i} \phi_{i}^{(n)}\right\rangle & =\sum_{i, j} k^{\prime} \int_{\{\hat{\rho} \in \mathcal{P}(\mathcal{H}) \mid \operatorname{tr} \hat{\rho} \rho>\beta\}}\left\langle\phi_{i}|\hat{\rho}| \phi_{j}\right\rangle^{n} \nu(d \hat{\rho}) \\
& =\frac{k^{\prime} \cdot(k-1) !}{\pi^{(k-1)}} \int_{0}^{\alpha} f_{1}\left(\theta_{1}\right) \cos \theta_{1} \sin ^{2 k-3} \theta_{1} d \theta_{1} \\
\left\langle\phi(0)^{(n)}\left|\hat{W}_{\beta}(\rho)\right| \phi(0)^{(n)}\right\rangle & =k^{\prime} \int_{\{\hat{\rho} \in \mathcal{P}(\mathcal{H}) \mid \operatorname{tr} \hat{\rho} \rho \leq \beta\}}\langle\phi(0)|\hat{\rho}| \phi(0)\rangle^{n} \nu(d \hat{\rho}) \\
& =C \int_{\alpha}^{\frac{\pi}{2}} \cos ^{2 n+1} \theta_{1} \sin ^{2 k-3} \theta_{1} d \theta_{1} \\
\left\langle\phi(0)^{(n)}\left|\operatorname{Id}-\hat{W}_{\beta}(\rho)\right| \phi(0)^{(n)}\right\rangle & =k^{\prime} \int_{\{\hat{\rho} \in \mathcal{P}(\mathcal{H}) \mid \operatorname{tr} \hat{\rho} \rho>\beta\}}^{\alpha}\langle\phi(0)|\hat{\rho}| \phi(0)\rangle^{n} \nu(d \hat{\rho}) \\
& =C \int_{0}^{\alpha} \cos ^{2 n+1} \theta_{1} \sin ^{2 k-3} \theta_{1} d \theta_{1} .
\end{aligned}
$$

Lemma A.4 The following function $f(x)$ is monotone decreasing on $[0, \infty)$ :

$f(x):=\sum_{a=1}^{m} \sum_{b=1}^{m} \underbrace{\int_{0}^{2 \pi} \cdots \int_{0}^{2 \pi}}_{k}\left(c_{a}^{0} e^{i d_{a}^{0}}+x \sum_{j=1}^{k} e^{i\left(\theta_{j}+d_{a}^{j}\right)} c_{a}^{j}\right)^{n}\left(c_{b}^{0} e^{i d_{b}^{0}}+x \sum_{j=1}^{k} e^{-i\left(\theta_{j}+d_{b}^{j}\right)} c_{b}^{j}\right)^{n} d \theta_{1} \cdots d \theta_{k}$.

where $c_{n}^{j}, d_{n}^{j}$ are any real numbers.

Proof The set $K_{n}^{m}$ is defined as follows:

$$
K_{n}^{m}:=\left\{I=\left(I_{1}, \cdot, I_{m}\right) \in\left(\mathbf{N}^{+, 0}\right)^{m} \mid \sum_{j=1}^{m} i_{j}=n\right\} .
$$

The number $C(I)$ is defined for $I \in K_{n}^{m}$ as sufficing the following condition:

$$
\left(\sum_{j=1}^{m} x_{j}\right)^{n}=\sum_{I \in K_{n}^{m}} C(I) x_{1}^{I_{0}} \ldots x_{m}^{I_{m}} .
$$

Therefore,

$$
\left(c_{a}^{0}+x \sum_{j=1}^{k} e^{i\left(\theta_{j}+d_{a}^{j}\right)} c_{a}^{j}\right)^{n}=\sum_{I \in K_{n}^{k+1}} C(I) e^{i d_{a}^{0}}\left(c_{a}^{0}\right)^{I_{0}} e^{i I_{1}\left(\theta_{1}+d_{a}^{1}\right)}\left(c_{a}^{1}\right)^{I_{1}} \ldots e^{i I_{d}\left(\theta_{k}+d_{a}^{k}\right)}\left(c_{a}^{k}\right)^{I_{k}} x^{n-I_{0}} .
$$

Thus,

$$
\begin{aligned}
& f(x) \\
= & \sum_{a=1}^{m} \sum_{b=1}^{m}(2 \pi)^{k} \sum_{I} C(I) e^{i I_{0}\left(d_{a}^{0}-d_{b}^{0}\right)}\left(c_{a}^{0} c_{b}^{0}\right)^{I_{0}} e^{i I_{1}\left(d_{a}^{1}-d_{b}^{1}\right)}\left(c_{a}^{1} c_{b}^{1}\right)^{I_{1}} \ldots e^{i I_{k}\left(d_{a}^{k}-d_{b}^{k}\right)}\left(c_{a}^{k} c_{b}^{k}\right)^{I_{k}} x^{2 n-2 I_{0}} \\
= & (2 \pi)^{k} \sum_{I} C(I) \sum_{a=1}^{m} \sum_{b=1}^{m} e^{i\left(\sum_{j=0}^{k} I_{i} d_{a}^{i}-\sum_{j=0}^{k} I_{i} d_{b}^{i}\right)}\left(c_{a}^{0}\right)^{I_{0}} \ldots\left(c_{a}^{k}\right)^{I_{k}}\left(c_{b}^{0}\right)^{I_{0}} \ldots\left(c_{b}^{k}\right)^{I_{k}} x^{2 n-2 I_{0}} \\
= & (2 \pi)^{k} \sum_{I} C(I) D(I) x^{2 n-2 I_{0}},
\end{aligned}
$$


where

$$
D(I):=\sum_{a=1}^{m} \sum_{b=1}^{m} e^{i\left(\sum_{j=0}^{k} I_{i} d_{a}^{i}-\sum_{j=0}^{k} I_{i} d_{b}^{i}\right)}\left(c_{a}^{0}\right)^{I_{0}} \ldots\left(c_{a}^{k}\right)^{I_{k}}\left(c_{b}^{0}\right)^{I_{0}} \ldots\left(c_{b}^{k}\right)^{I_{k}} .
$$

It is sufficient to show $D(I) \geq 0$. Letting

$$
\begin{aligned}
v_{a} & :=\left(c_{a}^{0}\right)^{I_{0}} \ldots\left(c_{a}^{k}\right)^{I_{k}} \\
y_{a} & :=\sum_{j=0}^{k} I_{i} d_{a}^{i} \\
w_{a, b} & :=\cos \left(y_{a}-y_{b}\right),
\end{aligned}
$$

we have

$$
D(I)=\sum_{a=1}^{m} \sum_{b=1}^{m} v_{a} w_{a, b} v_{b}
$$

Then

$$
w_{a, b}=\cos \left(y_{a}-y_{b}\right)=\cos y_{a} \cos y_{b}+\sin y_{a} \sin y_{b} .
$$

As $\left\{\cos y_{a} \cos y_{b}\right\}$ and $\left\{\sin y_{a} \sin y_{b}\right\}$ are nonnegative, $\left\{w_{a, b}\right\}$ is nonnegative matrix. Therefore, we obtain $D(I) \geq 0$.

\section{B Proof of Lemma 4.1}

$$
\begin{aligned}
\mathcal{D}^{W,(n)}\left(\Pi_{n}\right)= & \int_{\mathcal{P}(\mathcal{H})} h(\operatorname{tr} \rho \hat{\rho}) \operatorname{tr}\left(\Pi_{n}(d \hat{\rho}) \rho^{(n)}\right) \\
= & \int_{\mathcal{P}(\mathcal{H})} h\left(|\langle\phi(\theta) \mid \phi(0)\rangle|^{2}\right) k^{\prime}\left|\left\langle\phi(\theta)^{(n)} \mid \phi(0)^{(n)}\right\rangle\right|^{2} \nu(d \theta) \\
= & \int_{0}^{\frac{\pi}{2}} h\left(\cos ^{2} \theta_{1}\right) \frac{n H_{k}(k-1) !}{\pi^{k-1}} \cos ^{2 n+1} \theta_{1} \sin ^{2 k-3} \theta_{1} d \theta_{1} \\
& \times \underbrace{\int_{0}^{\frac{\pi}{2}} \cdots \int_{0}^{\frac{\pi}{2}}}_{k-2} \underbrace{\int_{0}^{2 \pi} \cdots \int_{0}^{2 \pi}}_{k-1} \sin ^{2 k-5} \theta_{2} \cdots \sin \theta_{k-1} \cos \theta_{2} \cdots \cos \theta_{k-1} d \theta_{2} \cdots d \theta_{2 k-2} \\
= & \int_{0}^{\frac{\pi}{2}} h\left(\cos ^{2} \theta_{1}\right) \frac{n H_{k}(k-1) !}{\pi^{k-1}} \cos ^{2 n+1} \theta_{1} \sin ^{2 k-3} \theta_{1} d \theta_{1} \\
& \times \underbrace{\int_{0}^{1} x^{2 k-5} d x \cdots \int_{0}^{1} x d x}_{k-2} \cdot(2 \pi)^{k-1} \\
= & \int_{0}^{\frac{\pi}{2}} h\left(\cos ^{2} \theta\right) \frac{n H_{k}(k-1) !}{\pi^{k-1}} \cos ^{2 n+1} \theta \sin ^{2 k-3} \theta d \theta \frac{(2 \pi)^{k-1}}{2^{k-2}(k-2) !} \\
= & 2(k-1){ }_{n} H_{k} \int_{0}^{\frac{\pi}{2}} h\left(\cos ^{2} \theta\right) \cos ^{2 n+1} \theta \sin ^{2 k-3} \theta d \theta .
\end{aligned}
$$

The proof is complete. 


\section{Proof of Lemma 5.1}

From the assumption, for any $\epsilon>0$, there exists $\delta>0$ such that for any $x \in[0, \delta]$

$$
g_{n}(x)(1-\epsilon) \leq f_{n}(x) \leq g_{n}(x)(1+\epsilon) .
$$

Therefore,

$$
(1-\epsilon) \int_{0}^{\delta} g_{n}(x) d x \leq \int_{0}^{\delta} f_{n}(x) d x \leq(1+\epsilon) \int_{0}^{\delta} g_{n}(x) d x .
$$

By the assumption, there exists $n_{0}$ such that for any $n \geq n_{0}$,

$$
\begin{aligned}
& \int_{\delta}^{a} f_{n}(x) d x \leq \epsilon \int_{0}^{a} f_{n}(x) d x \\
& \int_{\delta}^{a} g_{n}(x) d x \leq \epsilon \int_{0}^{a} g_{n}(x) d x .
\end{aligned}
$$

Thus,

$$
\begin{aligned}
(1-\epsilon) \int_{0}^{a} g_{n}(x) d x-(1-\epsilon) \epsilon \int_{0}^{a} g_{n}(x) d x & \leq \int_{0}^{\delta} f_{n}(x) d x \\
& \leq \int_{0}^{a} f_{n}(x) d x \\
& \leq(1+\epsilon) \int_{0}^{a} g_{n}(x) d x+\epsilon \int_{0}^{a} f_{n}(x) d x .
\end{aligned}
$$

We obtain

$$
\begin{gathered}
(1-\epsilon)^{2} \leq \frac{\int_{0}^{a} f_{n}(x) d x}{\int_{0}^{a} g_{n}(x) d x} \leq \frac{1+\epsilon}{1-\epsilon} . \\
(1-\epsilon)^{2} \leq \lim _{n \rightarrow \infty} \frac{\int_{0}^{a} f_{n}(x) d x}{\int_{0}^{a} g_{n}(x) d x} \leq \frac{1+\epsilon}{1-\epsilon} .
\end{gathered}
$$

Since $\epsilon$ is arbitrary, the proof is complete.

\section{References}

[1] P. Hausladen, R. Josa, B. Schumacher, M. Westmoreland, W. Wooters, "Classical information capacity of a quantum channel," Phys. Rev. A 54, 1869-1876 (1996).

[2] A. S. Holevo, "The Capacity of Quantum Channel with General Signal States," quant-ph/9611023 (1996).

[3] A. Ekert and P. Jozsa, "Quantum Computation and Shor's Factoring Algorithm," Review of Modern Physics, vol.68, No.3 pp.733, (1996).

[4] C. W. Helstrom, Quantum Detection and Estimation Theory, (Academic Press, New York, 1976). 
[5] A. S. Holevo, Probabilistic and Statistical Aspects of Quantum Theory, (North_Holland, Amsterdam, 1982).

[6] H. P. Yuen and M. Lax, "Multiple-parameter quantum estimation and measurement of nonselfadjoint observables," IEEE trans., IT-19, pp740-750 (1973).

[7] M. Hayashi, "A Linear Programming Approach to Attainable Cramér-Rao type Bounds and Randomness Condition," (submitting to Jour. Math. Phys.).

[8] M. Hayashi, "A Linear Programming Approach to Attainable Cramér-Rao type Bounds," in Quantum Communication, Computing, and Measurement, edited by O.Hirota, A.S.Holevo, and C.M.Caves, (Plenum Publishing, 1997) to appear .

[9] H. Nagaoka, "A generalization of the simultaneous diagonalization of hermitian matrices and its relation to quantum estimation theory," Trans. Jap. Soci. Ind. App. Math., vol.1, No.4, pp.305-318 (1991)(in Japanese).

[10] A. Fujiwara and H. Nagaoka, in Quantum coherence and decoherence, edited by K. Fujikawa and Y. A. Ono, (Elsevier, Amsterdam, 1996), pp. 303.

[11] K. Matsumoto, "A new approach to the Cramér-Rao type bound of the pure state model," METR 96-09,(1996).

[12] H. Nagaoka, "On the relation Kullback divergence and Fisher information -from classical systems to quantum systems-," (1992)(in Japanese).

[13] A. S. Holevo, "Covariant measurement and uncertainly relations," Reports on mathematical physics, vol.12, pp.251-271,(1977).

[14] P. Griffiths and J. Harris, Principle of Algebraic geometry, (John Wiley \& Sons, New York, 1978).

[15] G. W. Mackey, "Imprimitivity for representations of locally compact groups I" Proc. Nat. Acad. Sci. USA, vol. 35, pp.537-545,(1949).

[16] K. R. W. Jones, "Fundamental limits upon the measuremnt of state vectors", Phys. Rev. A, 50, 3682-3699 (1994). V. Bužek, G. Adam and G. Drobný, "Quntum state reconstructuin and detection of quantum coherences on deifferent observation levels", Phys. Rev. A 54 804-820 (1996). 